American Journal of Applied Sciences 5 (6): 626-632, 2008

ISSN 1546-9239

(C) 2008 Science Publications

\title{
Low-g Area-changed MEMS Accelerometer Using Bulk Silicon Technique
}

\author{
Badariah Bais and Burhanuddin Yeop Majlis \\ Institute of Microengineering and Nanoelectronics (IMEN), Universiti Kebangsaan Malaysia \\ 43600 UKM Bangi, Selangor, Malaysia
}

\begin{abstract}
A bulk micromachined accelerometer based on an area variation capacitive sensing for low-g applications was developed. The accelerometer was designed with ribbed-style fingers structure on the movable mass connected in parallel and suspended over stationary electrodes composed of differential comb fingers by means of suspension beams anchored onto the substrate. A folded, rigid truss suspension design with low spring constant and low cross-axis sensitivity was chosen. The simulation was performed using Coventorware software. A threemask bulk micromachining wafer bonding fabrication process was utilized to realize the accelerometer. Silicon-on-glass was used to achieve high sensitivity and low mechanical noise while maintaining a simple structure. The general concept, main design considerations, fabrication procedure and performance of the resulted accelerometer was elaborated and presented. A linear relationship between the differential capacitance and acceleration was obtained. The accelerometer sensitivity was calculated to be $0.47 \mathrm{pF} / \mathrm{g}$ with an acceleration range of $\pm 5 \mathrm{~g}$.
\end{abstract}

Key words: MEMS, area-changed, accelerometer, capacitive, bulk micromachined

\section{INTRODUCTION}

The interest in the development of low-g MEMS accelerometer has increased lately. Low-g accelerometer has potential applications in the automotive field such as vehicle stability enhancement, roll-over detection, inclination/theft detection and vehicle dynamics such as potential wheel skid while turning. Other applications include tilt, vibration and shock measurements ${ }^{1]}$. Low-g accelerometers are also developed for medical applications like detection of hand tremor or health monitoring ${ }^{[2-4]}$.

Majority of the low-g accelerometer were developed using the capacitive-sensing scheme wherein the deflection of the inertial mass produces a change in the capacitance that can then translated to electrical output $^{[5-6]}$. Low-g accelerometers require high sensitivity and low mechanical noise. High sensitivity is typically achievable by reducing the air gap between the sensing electrodes and increasing the overlap area between the electrode fingers. However, there are a few disadvantages with the structures having narrow air gaps and a large sensing area. Firstly, in accelerometers utilizing varying air-gap, the deflection of the proof mass produces a change in the air gap between electrodes that causes the change in capacitance. Since capacitance is inversely proportional to the square of the air gap, there is an inherent nonlinearity from the varying air-gap structure. Secondly, since the movement of the proof mass must also be limited in a small range in order to improve the linearity, the differential capacitance tends to be very small. This in turn makes the design of the detection circuit difficult ${ }^{[7]}$.

Area-changed type of capacitance detection scheme provides an attractive alternative approach to overcome the disadvantages arising from the air-gap change type of accelerometer. Area-changed accelerometers uses a ribbed fingers structure on the movable mass as a differential capacitor and suspended over stationary electrodes composed of differential comb fingers by means of suspension beams ${ }^{[8-10]}$. With the arrangement of the sensing electrodes on top of another, the differential capacitance is shown to vary linearly with the overlapped area of the proof mass and the fixed electrodes underneath and independent of the gap between the proof mass and the electrodes. Also, as the sense electrodes move coplanar with respect to each

\section{Corresponding Author: Badariah Bais, Institute of Microengineering and Nanoelectronics (IMEN), Universiti} Kebangsaan Malaysia, Malaysia 
other, the operational range of the device is not limited the thickness of air gap.

High sensitivity can be obtained either by increasing the mass of the proof mass or lowering the spring constant of the suspension beam. The mass of the proof mass can be increased by increasing the thickness and the size of the proof mass. Folded suspension designs can be used to achieve high sensitivity by offering a low spring constant in the sensing direction. It is also desired that the suspension is compliant in the direction of displacement and very stiff in the orthogonal directions. With this approach, the sensitive axis is parallel to the air gap of the sense fingers and therefore the pull-in voltage of the device is increased considerably. Lastly, squeeze film damping between the sense fingers is avoided as there is no movement towards one another.

Surface micromachining technology is very attractive for use in various applications because of its compatibility with the IC processes and the size of the device is small compared with those fabricated by the bulk micromachining ${ }^{[11-12]}$. However, the thickness of surface-micromachined accelerometers is limited by fabrication constraints. This makes the sensing mass to be very small which results in low sensitivity and high thermal mechanical noise. Bulk micromachined siliconon-glass accelerometers utilizing DRIE technology was developed in order to overcome the small sensing mass [13-14]. Compared with surface-micromachining technology, bulk micromachining technology is a very promising approach to fabricate these accelerometers as its thickness can be made as thick as necessary in order to meet its performance requirements.

In this study, the accelerometer structure and design is presented. The relevant analytical understanding for the mechanical behaviour of the sensing elements is discussed. The design analysis is verified by both electrostatic and mechanical simulation of the sensing element structures obtained through the Finite-Element Analysis (FEA). Bulk micromachining technique is chosen to be the fabrication technology for the accelerometer. A three-mask fabrication process is developed to realize the accelerometer utilizing silicon to glass anodic bonding and Deep Reactive Ion Etching (DRIE) in order to achieve a large proof mass and low mechanical noise device. The fabricated devices are then tested and measured.

\section{ACCELEROMETER STRUCTURE AND DESIGN}

The accelerometer used in this study as shown in Fig. 1 is based on the design by B. Li et al., which uses the area-changed principle of capacitive detection

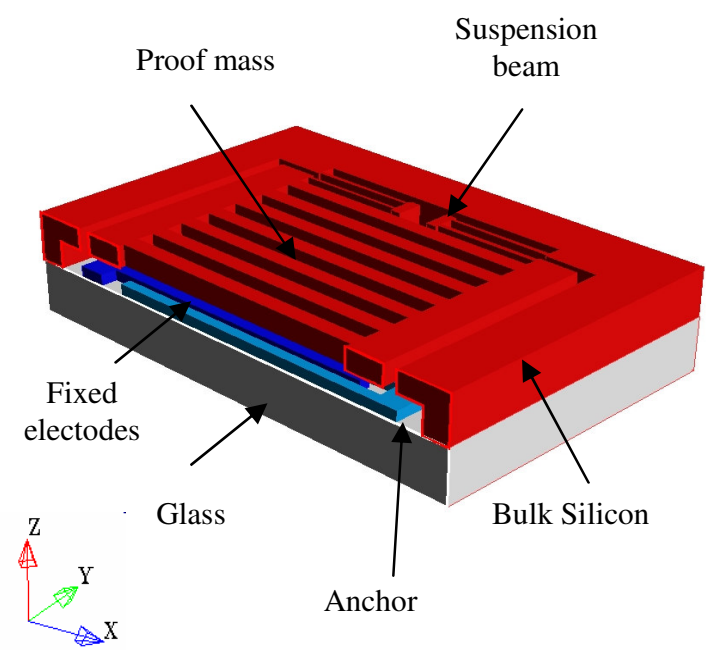

Fig. 1: 3D view of the area-changed accelerometer

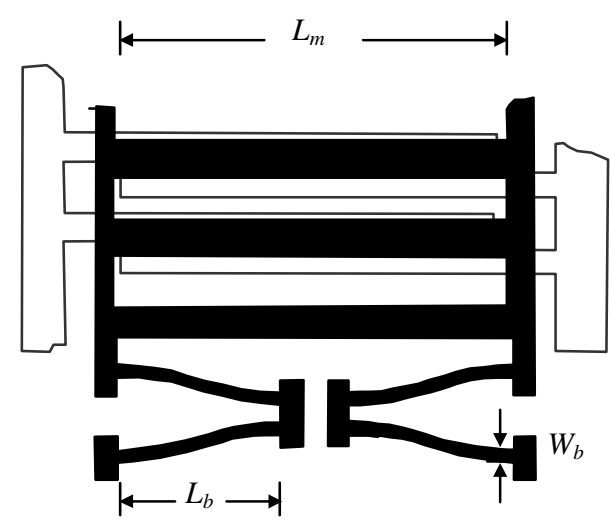

Fig. 3: Working principle of the accelerometer (a) no acceleration (b) with acceleration

scheme $^{[8]}$. It consists of a $40 \mu \mathrm{m}$ thick silicon proof mass suspended $3 \mu \mathrm{m}$ above the glass substrate by means of folded suspension beams that are anchored onto the substrate. The proof mass consists of many fingers where each of the fingers of the proof mass are $1600 \mu \mathrm{m}$ long connected in parallel. Stationary electrodes composed of differential comb fingers are placed directly under the mass. Under acceleration, the mass will move along the $y$ direction parallel to the substrate.

Figure 2 shows the top view of the area-changed accelerometer while Fig. 3 illustrates the principle of operation of the capacitive accelerometer structure. If there is no acceleration on the device, the capacitance $C_{A}$ and $C_{B}$ is the same as shown in Fig 3a. When acceleration is applied to the proof mass, the 


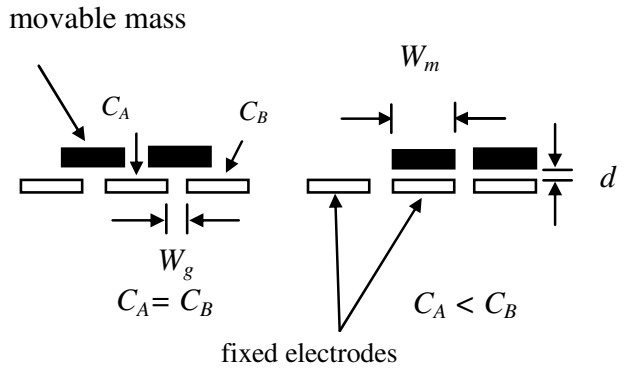

(a)

(b)

Fig. 3: Working principle of the accelerometer (a) no acceleration (b) with acceleration

accelerometer will move resulting in a change in capacitance as shown in Fig. $3 b$.

The accelerometer can be modeled as a lumped mass-spring-damper system. The dynamic behaviour of this lumped element system can be described by the second-order dynamic differential equation of motion where the displacement, $\Delta y_{m}$ as a function of force generated by the acceleration, $\mathrm{F}$ is given by the following:

$$
\mathrm{F}=\mathrm{ma}=\mathrm{m} \frac{\mathrm{d}^{2} \Delta \mathrm{y}_{\mathrm{m}}}{\mathrm{dt}^{2}}+\mathrm{b} \frac{\mathrm{d} \Delta \mathrm{y}_{\mathrm{m}}}{\mathrm{dt}}+\mathrm{k} \Delta \mathrm{y}_{\mathrm{m}}
$$

where, $\mathrm{m}$ is the mass of the proof mass, $\mathrm{a}$ is the applied acceleration, $\mathrm{b}$ is the damping coefficient and $\mathrm{k}$ is the spring constant of the mechanical suspension. When acceleration, a is applied to the accelerometer, the proof mass exerts a force, $F$ on the suspension. For frequencies below the mechanical resonance of the mass-spring system, this force causes the suspension to deflect a distance, $\Delta \mathrm{y}_{\mathrm{m}}$ and a linear relationship in (1) can be assumed as:

$$
\frac{\Delta \mathrm{y}_{\mathrm{m}}}{\mathrm{a}}=\frac{\mathrm{m}}{\mathrm{k}}
$$

The undamped resonant frequency is given by:

$$
\omega_{\mathrm{o}}=\sqrt{\frac{\mathrm{k}}{\mathrm{m}}}
$$

where, $\omega_{0}$ is the natural frequency of the accelerometer in the direction of applied acceleration. From Eq. 2, it is seen that the rate of change of displacement is proportional to the mass of the proof mass and inversely proportional to the spring constant for a given acceleration. Therefore, a higher rate of displacement of the proof mass per $\mathrm{g}$ is obtained by using a heavier proof mass and a reduced spring constant of the suspension beam. Hence, a smaller spring constant is needed to improve the device mechanical sensitivity. However, from Eq. 3, lowering the spring constant will result in a lower resonance frequency. This is the tradeoff in performance of the accelerometer.

The sensitivity of an accelerometer is determined by its capacitive sensitivity $\left(\mathrm{F} \mu \mathrm{m}^{-1}\right)$ and mechanical sensitivity $\left(\mu \mathrm{m} \mathrm{g}^{1}\right)$. In this analysis, the width of the proof mass bars, $\mathrm{W}_{\mathrm{m}}$, is set to be the same as the width of the fixed electrode fingers and the length of the mass bars, $L_{m}$, be the same as the length of the fixed electrode fingers under the mass bars. The vertical distance between the mass and the fixed electrodes is set as $\mathrm{d}$. Therefore, the static capacitance, $\mathrm{C}_{\mathrm{S}}$ is written as:

$$
\mathrm{C}_{\mathrm{A}}=\mathrm{C}_{\mathrm{B}}=\mathrm{C}_{\mathrm{S}}=\mathrm{N} \frac{\varepsilon \mathrm{L}_{\mathrm{m}}\left(\mathrm{W}_{\mathrm{m}}-\mathrm{W}_{\mathrm{g}}\right)}{2 \mathrm{~d}}
$$

where, $\mathrm{N}$ is the number of the fingers in the proof mass, $\varepsilon$ is the permittivity and $\mathrm{W}_{\mathrm{g}}$ is the gap between the fixed electrodes. Under applied acceleration, the changed capacitance $\mathrm{C}_{\mathrm{A}}$ and $\mathrm{C}_{\mathrm{B}}$ are as follows:

$$
\begin{aligned}
& \mathrm{C}_{\mathrm{A}}=\mathrm{N} \frac{\varepsilon\left(\mathrm{W}_{\mathrm{m}}-\mathrm{W}_{\mathrm{g}}+2 \Delta \mathrm{y}_{\mathrm{m}}\right) \mathrm{L}_{\mathrm{m}}}{2 \mathrm{~d}} \\
& \mathrm{C}_{\mathrm{B}}=\mathrm{N} \frac{\varepsilon\left(\mathrm{W}_{\mathrm{m}}-\mathrm{W}_{\mathrm{g}}+2 \Delta \mathrm{y}_{\mathrm{m}}\right) \mathrm{L}_{\mathrm{m}}}{2 \mathrm{~d}}
\end{aligned}
$$

Therefore, the differential capacitance can be written as below:

$$
\Delta \mathrm{C}=\mathrm{C}_{\mathrm{A}}-\mathrm{C}_{\mathrm{B}}=\frac{N \varepsilon \mathrm{L}_{\mathrm{m}}}{2 \mathrm{~d}}\left(4 \Delta \mathrm{y}_{\mathrm{m}}\right)
$$

Hence, the sensitivity of the accelerometer can be expressed as:

$$
\operatorname{Sens}(\mathrm{pF} / \mathrm{g})=\frac{\Delta \mathrm{C}}{\Delta \mathrm{y}_{\mathrm{m}}} \cdot \frac{\Delta \mathrm{y}_{\mathrm{m}}}{\mathrm{g}}=9.8 \frac{4 \mathrm{C}_{\mathrm{s}}}{\left(\mathrm{W}_{\mathrm{m}}-\mathrm{W}_{\mathrm{g}}\right)} \frac{\mathrm{m}}{\mathrm{k}}
$$

Folded suspensions with straight truss are employed in the design and are attached to each end of the movable proof mass and can be made very compliant. This design also provides low cross-axis sensitivity. Analysis shows the cross-axis sensitivity to 
be less than $0.7 \%^{[15]}$. Mechanical noise can be reduced by increasing the size of the proof mass.

The minimum detectable signal is mainly determined by the mechanical noise which can be reduced by increasing the size of the proof mass. The theoretical minimum detectable signal, $\mathrm{a}_{\min }$, due to the Brownian motion ${ }^{[16]}$ can be expressed as follows:

$$
\mathrm{a}_{\min }=\sqrt{\frac{4 \mathrm{k}_{\mathrm{b}} \mathrm{T} \omega_{\mathrm{o}}}{\mathrm{mQ}}}
$$

where; $\mathrm{k}_{\mathrm{b}}$ is the Boltzmann constant, $\mathrm{T}$ the absolute temperature, $\omega_{0}$ the resonant frequency, $\mathrm{m}$ is the mass of the proof mass and $\mathrm{Q}$ is the quality factor of the accelerometer.

In order to obtain a high sensitivity and low mechanical noise, the proof mass should be as large as possible ${ }^{[17]}$. Large proof mass can be achieved by increasing the thickness and/or increasing the surface area which will increase the capacitance value. However, the thickness of the proof mass can only be increased up to the limitation of the DRIE capability of the fabrication centre in order to obtain vertical etching profiles of the proof mass fingers. Therefore, the design of the accelerometer needs to be optimized to achieve high sensitivity and minimum mechanical noise based on the allowable fabrication process parameters. In this study, the thickness of the proof mass is limited to a maximum of $40 \mu \mathrm{m}$ and the minimum dimension of the proof mass and suspension beam widths and gap are fixed to $2 \mu \mathrm{m}$. The accelerometer is to be operating in the range of $\pm 5 \mathrm{~g}$. The design parameters are then optimized by using Excel Solver ${ }^{\circledR}$ under the constraint parameters and specifications of the accelerometer. Bulk silicon is chosen to be the material for the proof mass with density, $\rho=2.33 \times 10^{-3} \mathrm{~kg} \mathrm{~m}^{-3}$ and Young's modulus, $\mathrm{E}=1.72 \times 10^{11} \mathrm{~N} \mathrm{~m}^{-2}$.

\section{RESULTS AND DISCUSSION}

In this section, the electrostatic and mechanical simulation of the sensing structures was carried out and compared with the analytical results. Three types of the accelerometer was fabricated and tested.

Electrostatic behaviour: Simulations are carried out using Coventorware software. Figure 4 depicts the results of analytical and simulation of the displacement due to acceleration. From the plot, it can be shown that the rate of displacement is $0.064 \mu \mathrm{m} \mathrm{g}^{-1}$ from theoretical calculation and $0.076 \mu \mathrm{m} \mathrm{g}^{-1}$ from the simulation. It was calculated that the deviation from the

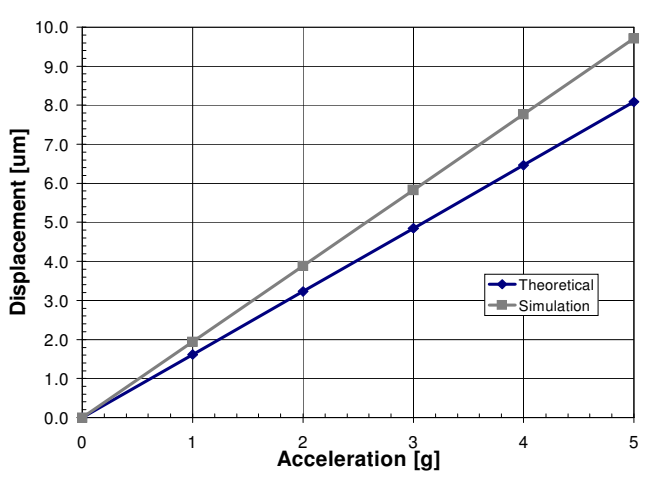

Fig. 4: Comparison of calculated and simulated displacement as a function of acceleration

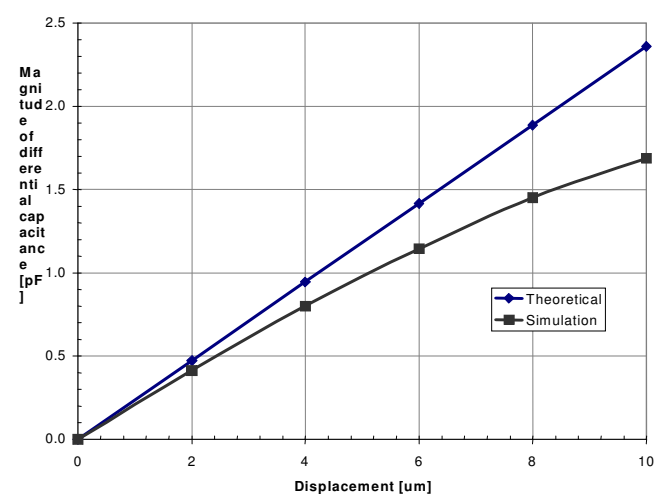

Fig. 5: Variation of differential capacitance as a function of lateral displacement

analytic expression in Eq. 4 and the simulation results was less than $18 \%$.

The calculated static capacitance for the accelerometer is $1.18 \mathrm{pF}$ while the simulated value is $2.33 \mathrm{pF}$. The magnitude of the differential capacitance variation as a function of lateral displacement is plotted in Fig. 5. From Fig. 5, it is seen that the simulation results deviate from the theoretical results by $18 \%$ as the displacement increases. This is due to the increased in parasitic and fringe capacitances with displacement.

Mechanical behaviour: Finite-Element Analysis (FEA) was performed using Coventorware in order to calculate the resonance frequency and the sensitivity including cross-axis of the sensor element. Structural as well as harmonic analysis were performed in order to obtain the data. Figure 6 shows the mode shapes of the accelerometer under the effect of a $5 \mathrm{~g}$ acceleration in each of the axes $x, y$ and $z$. Simulation results show that the most sensitive as well as the lowest frequency was 


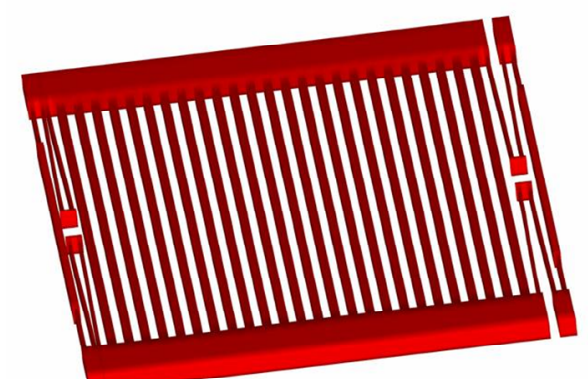

(a)

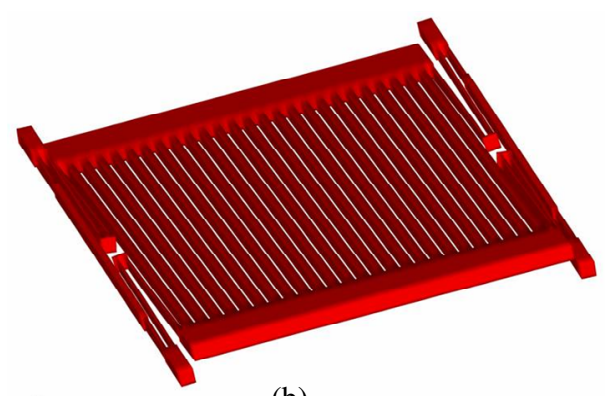

(b)

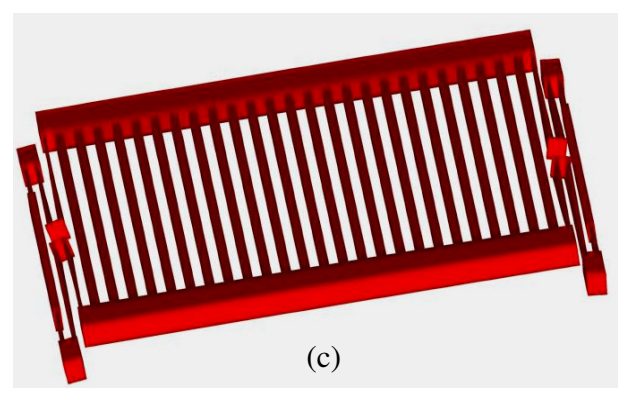

Fig. 6: Mode shapes of the proof mass of the accelerometer (a) first mode (b) second mode (c) third mode

in the y-axis, followed by the rotation of the mass due to cross-axis in the y-direction and lastly due to acceleration in the $\mathrm{z}$-axis. The first mode resonant frequency is at $365 \mathrm{~Hz}$ while the second and third modes are at 3.0 and $3.4 \mathrm{kHz}$, respectively. Since the second and the third modes are much higher than the first mode, low cross-axis sensitivity is expected.

Figure 7 shows the plot of the sensitivity of the accelerometer as a function of displacement. It is seen that the simulated results deviate from the calculated values as the displacement increases. The deviation in the simulated sensitivity of the device was primarily due to the parasitic capacitance and fringe capacitance. It can be shown that the simulated sensitivity of the

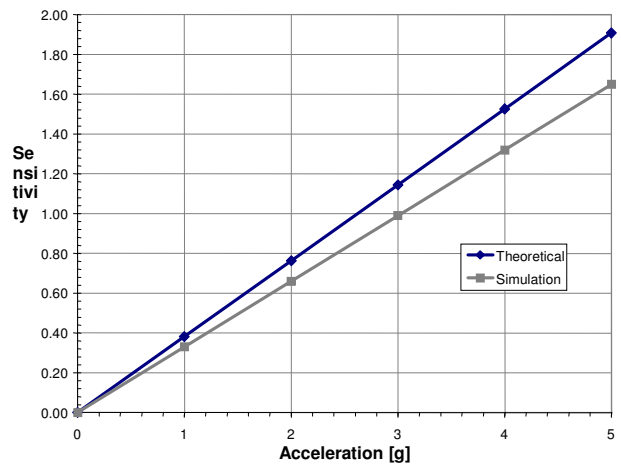

Fig. 7: Sensitivity of the accelerometer as a function of acceleration

Table 1:The calculated performance of the area-changed accelerometer

\begin{tabular}{|c|c|c|c|}
\hline Specification & Type1 & Type2 & Type3 \\
\hline Proof mass length $(\mu \mathrm{m})$ & 1600 & 1600 & 1600 \\
\hline Proof mass width $(\mu \mathrm{m})$ & 22 & 6 & 22 \\
\hline Movable mass $(\mu \mathrm{g})$ & 128 & 41 & 128 \\
\hline Suspension beam length $(\mu \mathrm{m})$ & 560 & 566 & 560 \\
\hline Suspension beam width $(\mu \mathrm{m})$ & 2 & 2.3 & 3 \\
\hline Thickness of proof mass $(\mu \mathrm{m})$ & 40 & 40 & 40 \\
\hline Spring constant $\left(\mathrm{N} \mathrm{m}^{-1}\right)$ & 0.63 & 0.92 & 2.12 \\
\hline Resonant frequency $(\mathrm{Hz})$ & 350 & 760 & 650 \\
\hline Rest capacitance $(\mathrm{fF})$ & 1180 & 236 & 1180 \\
\hline Sensitivity ( $\mathrm{fF}^{-1}$ ) & 474 & 103 & 141 \\
\hline Range & $\pm 5 \mathrm{~g}$ & $\pm 5 \mathrm{~g}$ & $\pm 5 \mathrm{~g}$ \\
\hline Mechanical noise floor $(\mu \mathrm{g} / \sqrt{ } \mathrm{Hz})$ & 0.3 & 0.53 & 0.3 \\
\hline
\end{tabular}

device is lower than that of the theoretical sensitivity of about 58\%. However, its linearity agrees. The calculated performance of the resulted accelerometer is shown in Table 1.

Fabrication and testing: The area-changed accelerometer was fabricated with three masks bulk micromachining fabrication process in order to define the main design structures namely one on the glass to form the fixed electrodes and metal interconnects and two on the silicon to create the cavity and to define the sensor structure and electrodes.

The main fabrication process consists of different modules such as glass processing, silicon processing, anodic bonding of the two substrates, Chemical Mechanical Polishing (CMP) and Deep Reactive Ion Etching (DRIE). The sequence of the fabrication process is shown in Fig. 6.4. First, the glass wafer is initially cleaned and prepared for deposition of the $\mathrm{Cr} / \mathrm{Au}$. Next, the metal is patterned to define the fixed electrodes structure and lower metal contacts. A cavity of $3 \mu \mathrm{m}$ is then formed in the silicon substrate. Following that the glass wafer and silicon are 


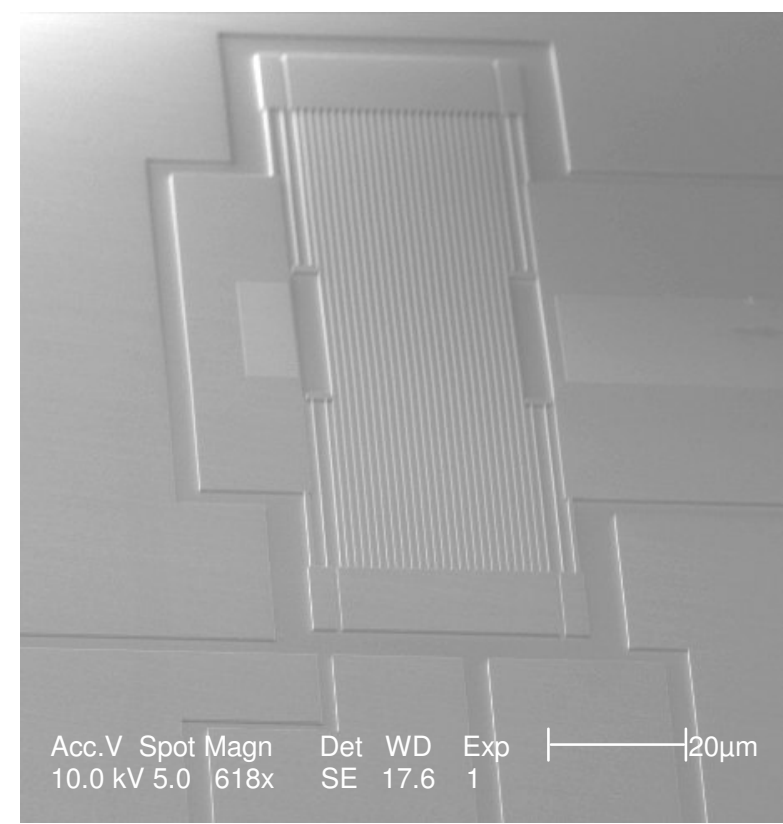

Fig. 8: SEM micrograph of a type 2 area-changed accelerometer

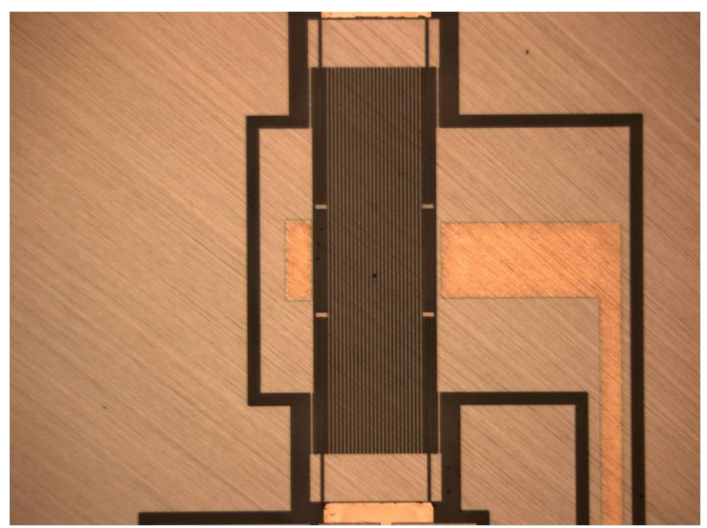

Fig. 9: Optical micrograph of the proof mass of the area-changed accelerometer

anodically bonded. After bonding, the silicon wafer is thinned to the desired thickness of $40 \mu \mathrm{m}$ by using standard CMP. After the CMP step, the metal contacts of $\mathrm{Al}$ are sputtered and patterned. Finally, the movable proof mass structure is patterned using Deep Reactive Ion Etching (DRIE).

Figure 8 shows the SEM micrograph of a typical device. A folded suspension beam can be seen along with movable proof mass sensing fingers.

Figure 9 shows the optical micrograph of fabricated device of the area-changed accelerometer

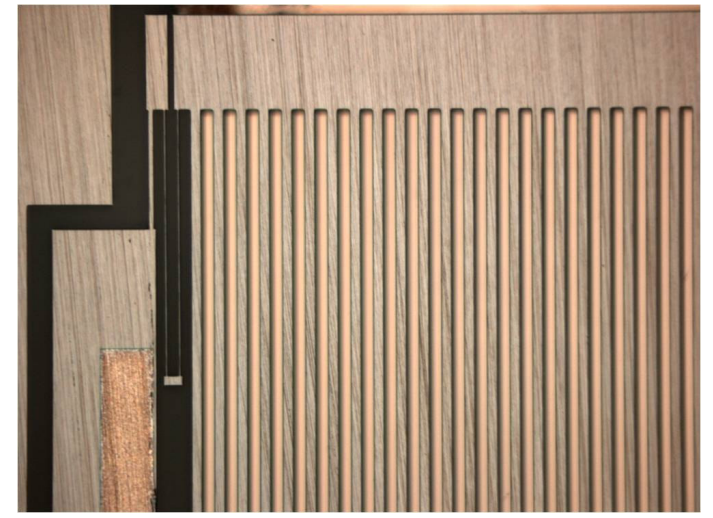

Fig. 10: Close-up of the folded suspension beams and the proof mass fingers

while Fig. 10 shows the close-up view of the folded suspension beams and the proof mass structure of the accelerometer.

From the measurement made on the geometrical structures of the fabricated proof mass and the fixed electrodes, it was found that the designed dimensions agree well with the measured ones with the highest deviation of $3.38 \%$. The static capacitance of the fabricated devices is measured using Agilent 4284A precision LCR meter. The highest deviation between the measured and the simulated static capacitances is about $10 \%$.

\section{CONCLUSION}

Design, simulation and performance evaluation of a bulk-micromachined area-changed capacitive accelerometer for low-g applications was presented. Theoretical modeling was performed and verified with the simulation results. A folded suspension design with rigid truss that offers low spring constant and low cross-axis sensitivity was chosen. Various parameters were studied and optimized with Excel Solver® in order to achieve high sensitivity and low mechanical noise. A three-mask bulk micromachining wafer bonding fabrication process was utilized to produce this accelerometer. Silicon-on-glass was used to achieve low mechanical noise with high sensitivity while maintaining a simple structure. The resulted accelerometer was $40 \mu \mathrm{m}$ thick and $2.2 \times 1.5 \mathrm{~mm}$ in size with a calculated sensitivity is up to $0.47 \mathrm{pF} \mathrm{g}^{-1}$. The calculated mechanical noise was $0.3 \mu \mathrm{g} / \sqrt{ } \mathrm{Hz}$ at atmospheric pressure. The deviations between the measured and simulated values for the geometrical 
variations and static capacitance are 3.38 and $10 \%$, respectively.

\section{ACKNOWLEDGMENT}

This project is financially supported by Ministry of Science, Technology and Environment of Malaysia under IRPA program: Development of MEMS Technology for Automotive Application.

\section{REFERENCES}

1. Yazdi, N., K. Najafi and A.S. Salian, 2003. A High-Sensitivity Silicon Accelerometer with a Folded-Electrode Structure. J. Microelectromechanical Syst., 12 (4): 479-486.

2. Graham, B.B., 2000. Using an accelerometer sensor to measure human hand motion, $\mathrm{PhD}$. Thesis, Massachusetts Institute of Technology.

3. Biefeld, V., B. Clasbrummel and J. Binder, 2000. Implantable low-g accelerometer for the telemetric monitoring of micro-movements in fracture zones. Proc. 1st Annual Int. IEEE-EMBS Special Topic Conf. on Microtechnologies in Medicine and Biology, pp: 497-501.

4. Lötters, J.C., W. Olthuis, P.H. Veltink and P. Bergveld, 1997. Design, realization and characterization of a symmetrical triaxial capacitive accelerometer for medical applications. Sensors Actuators, A61: 303-308.

5. Henrion, W., L. DiSanza, M. Ip, S. Terry and H. Jerman, 1990. Wide Dynamic Range Direct Digital Accelerometer. 4th Tech. Dig. IEEE Solid-State Sensors and Actuator Workshop, pp: 153-157.

6. Chau, K.H.-L., S.R. Lewis, Y. Zhao, R.T. Howe, S.F. Bart and R.G. Marcheselli, 1996. Integrated Force-balanced Capacitive Accelerometer for Low-g Applications. Sensors and Actuators A54: 472-476.

7. Selvakumar, A., 1997. A Multifunctional Silicon Micromachining Technology for High Performance Microsensor and Microactuators, $\mathrm{PhD}$. Thesis, University of Michigan.

8. Li, B., D. Lu and W. Wang, 2001. Micromachined Accelerometer with Area-changed Capacitance. Mechatronics, 11: 811-819.

9. Ha, B., Y. Oh and C. Song, 1998. A Capacitive Silicon Microaccelerometer with ForceBalancing Electrodes. Jpn. J. Applied Physics., 37: 7052-7057.
10. Selvakumar, A. and K. Najafi, 1998. A High-Sensitivity Z-Axis Capacitive Silicon Microaccelerometer with a Torsional Suspension. J. Microelectromechanical Systems, 7 (2): 192-200.

11. Van Drieënhuizen, B.P., N.I. Maluf, I.E. Opris and G.T.A. Kovacs, 1997. Force-Balanced Accelerometer with $\mathrm{mG}$ Resolution. Fabricated using Silicon Fusion Bonding and Deep Reactive Ion Etching. 1997 Int'l. Conf. Solid-State Sensors and Actuators, Chicago, June 16-19, pp: 1229-1230.

12. Lu, C., M. Lemkin and B. Boser, 1995. A Monolithic Surface Micromachined Accelerometer with Digital Output. IEEE J. Solid State Circuits, 30 (12): 1367-1373.

13. Chae, J., H. Kulah and K. Najafi, 2002. A Hybrid Silicon-on-Glass (SOG) Lateral MicroAccelerometer with CMOS Readout Circuitry. Proc. 15th Int'l Conf. on MicroElectroMechanical Systems (MEMS2002), Las Vegas, NV., January 2002, pp: 623-626.

14. Xiao, Z., M. Chen, G. Wu, C. Zhao, D. Zhang, Y. Hao, G. Zhang and Z. Li, 1999. Laterally Capacity Sensed Accelerometer Fabricated with Anodic Bonding and High Aspect Ratio Etching. Proc. 10th Int'l Conf. Solid-State Sensors and Actuators (TRANSDUCERS '99), Sendai, Japan, pp: 1518-1521.

15. Badariah Bais and Burhanuddin Yeop Majlis, 2004. Suspension Design Analysis on the Performance of MEMS Area-changed Lateral Capacitive Accelerometer. Proc. 2004 IEEE Int'1 Conf. on Semiconductor Electronics (ICSE2004), Kuala Lumpur, Malaysia, pp: 335-339.

16. Gabrielson, T.B., 1993. Mechanical-thermal Noise in Micromachined Acoustic and Vibration Sensors, IEEE Transactions on Electron Devices, 40 (5): 903-909.

17. Yazdi, N. and K. Najafi, 2000. An All-silicon Single-wafer Micro-g Accelerometer with a Combined Surface and Bulk Micromachining Process. J. Microelectromechanical Systems, 9 (4): 544-550. 